\title{
Demonstration of a High Average Power Tabletop Soft X-Ray Laser
}

\author{
B. R. Benware, C. D. Macchietto, C. H. Moreno, and J. J. Rocca \\ Department of Electrical Engineering, Colorado State University, Fort Collins, Colorado 80523
}

(Received 31 August 1998; revised manuscript received 9 November 1998)

\begin{abstract}
We report the first demonstration of a high average power tabletop soft $\mathrm{x}$-ray laser. An average laser output power of $\approx 1 \mathrm{~mW}\left(>2 \times 10^{14}\right.$ photons/s) was generated at $46.9 \mathrm{~nm}$ in Ne-like Ar using a very compact tabletop discharge. The spatially coherent average power emitted by this $26.5 \mathrm{eV}$ laser is comparable to that generated at this photon energy in a similar bandwidth $\left(\Delta \lambda / \lambda=10^{-4}\right)$ by a thirdgeneration synchrotron beam line. Lasing was obtained at a repetition rate of $7 \mathrm{~Hz}$ with an average output energy of $135 \mu \mathrm{J} /$ pulse by exciting a plasma column in a ceramic capillary with a fast current pulse. This very compact high-repetition-rate laser source makes intense short-wavelength coherent radiation accessible to a wide variety of new applications. [S0031-9007(98)08022-3]

PACS numbers: $42.55 . \mathrm{Vc}, 52.55 . \mathrm{Ez}, 52.75 . \mathrm{Va}$
\end{abstract}

Important motivations for the development of high average power tabletop sources of coherent soft x-ray radiation can be found in applications belonging to several disciplines of science and technology. To date, the leading sources of high average power coherent soft x-ray radiation have been undulators in modern storage rings, so-called third-generation synchrotrons, in which radiation is emitted by a highly relativistic electron beam as it traverses a periodic magnetic structure [1]. Synchrotrons are used successfully in many areas including material surface diagnostics, photochemistry and photophysics, atomic and molecular physics, very highresolution metrology, and biology. They have the important advantages of having a high average brightness and a broad tunability. However, they are typically large facilities that are very expensive to construct and operate.

High average power beams of soft $\mathrm{x}$-ray coherent radiation can in principle also be generated with much more compact devices using either nonlinear optical techniques for frequency up-conversion of optical laser radiation or the direct amplification of spontaneous emission in a plasma (an x-ray laser). At present, the generation of high order harmonics under fairly optimized nonphased matched conditions typically yields a conversion efficiency of about $10^{-6}$ in the photon energy range of $10-40 \mathrm{eV}$ (of the order of $10^{9}$ photons per pulse) [2]. The highest pulse energy generated in a high order harmonic pulse to date is $60 \mathrm{~nJ}$ at a photon energy of about $50 \mathrm{eV}$, and corresponds to an experiment done using the second harmonic of a powerful Nd-glass laser system [3]. Recently, a very important advance in this field was accomplished with the first demonstration of phase-matched harmonic conversion of visible light into soft $\mathrm{x}$ rays [4]. A conversion efficiency of $10^{-5}-10^{-6}$ was obtained in the $40-70 \mathrm{eV}$ spectral region. Soft $\mathrm{x}$-ray pulses with an energy of $>0.2 \mathrm{~nJ}$ per harmonic order were produced at a repetition frequency of $1 \mathrm{kHz}$, corresponding to an average power of $>0.2 \mu \mathrm{W}$ [4]. This result is significant because further optimization of this technique could result in the generation of high average power beams of spatially coherent soft x-ray radiation from a tabletop optical laser.

Alternatively, soft $\mathrm{x}$-ray lasers have the advantage of a much higher energy per pulse and therefore the potential to produce soft $\mathrm{x}$-ray beams with very high average powers. X-ray lasers pumped by large optical lasers of the type used in fusion research have generated output pulse energies of up to several $\mathrm{mJ}$ [5]. However, these lasers are limited to a repetition frequency of only a few pulses per hour at best. Recently, significant progress has been accomplished in the development of more compact soft $\mathrm{x}$-ray lasers that can operate at increased repetition frequencies [6-11]. Soft x-ray lasers based on the transient collisional excitation scheme, which are pumped by picosecond lasers that typically occupy a few optical tables, have produced soft x-ray amplification at repetition rates up to one shot every 3 min [6,7]. Our group has previously reported the generation of laser output pulse energies up to $25 \mu \mathrm{J}$ in the $J=0-1$ line of $\mathrm{Ne}$-like Ar at $46.9 \mathrm{~nm}$ from single pass amplification in a compact capillary discharge tabletop soft x-ray amplifier [11]. However, in all these lasers the average power obtained to date has been very low, as a result of the low repetition rate ( $\leq 2$ shots per min). In contrast, soft $\mathrm{x}$-ray amplification at high repetition rates has been obtained utilizing relatively compact high intensity ultrashort pulse lasers to pump soft x-ray lasers in plasmas created by optical-field-induced ionization [8,9]. Amplification was demonstrated at $10 \mathrm{~Hz}$ at $41.8 \mathrm{~nm}$ in collisionally excited Pd-like $\mathrm{Xe}$ (11 to 12 gain-lengths) [8], and at $2 \mathrm{~Hz}$ at $13.5 \mathrm{~nm}$ in $\mathrm{H}$-like Li following plasma recombination ( $\approx 5.5$ gain-lengths) [9]. Similar gain was obtained at $1 \mathrm{~Hz}$ at $26.2 \mathrm{~nm}$ in $\mathrm{H}$-like $\mathrm{B}$ in a recombining plasma excited by the combination of two low power laser pulses [10]. However, the average power obtained in these laserpumped soft x-ray amplifier systems was small due to the low laser output pulse energy, and required the use of high-gain microchannel plates to detect the laser line. An important next step in the development of practical tabletop soft $\mathrm{x}$-ray lasers is the generation of high average 
output powers, which simultaneously requires a high repetition rate and a high energy per pulse. In this Letter we report the first demonstration of a high average power tabletop soft x-ray laser. An average power of $0.95 \mathrm{~mW}$ was generated at $46.9 \mathrm{~nm}$. This value exceeds the average powers obtained to date with tabletop soft x-ray lasers by 2 to 3 orders of magnitude. Our results were obtained by generating an axially uniform plasma column with a fast current pulse at a repetition rate of $7 \mathrm{~Hz}$ in an alumina $\left(\mathrm{Al}_{2} \mathrm{O}_{3}\right)$ capillary filled with preionized $\mathrm{Ar}$ gas. The capillary discharge-pumped laser used in this experiment occupies an area of approximately $0.4 \mathrm{~m} \times 1 \mathrm{~m}$ on top of a table, a size comparable to that of many widely utilized visible or ultraviolet gas lasers.

In capillary discharges the amount of material ablated from the walls by plasma radiation and electron heat conduction can greatly affect the plasma compression and heating. In the polyacetal capillaries utilized in our previous discharge-pumped collisional soft $\mathrm{x}$-ray laser experiments $20 \%$ to $50 \%$ of the $\approx 39 \mathrm{kA}$ discharge current necessary to excite Ne-like Ar is computed to flow through material ablated from the walls [12]. This significantly reduces the efficiency of the plasma compression. In the present experiment, we utilized $\mathrm{Al}_{2} \mathrm{O}_{3}$ capillaries that are much more resistant to ablation and also have a larger heat conductivity that allows for the dissipation of high average discharge powers. An indication of the dramatic reduction in the mass of ablated material in the ceramic capillaries as compared to the polyacetal capillaries is the much smaller pressure increase measured at the exit of the capillary channel following a discharge shot: $<10$ mTorr instead of several Torr. This reduced wall ablation in ceramic capillaries results in a more efficient use of the current pulse, which allows for a significantly lower excitation current and for operation at high repetition rates. Lasing with an average power of $0.95 \mathrm{~mW}$ was obtained by exciting Ar filled alumina capillaries $3.2 \mathrm{~mm}$ in diameter and $18.2 \mathrm{~cm}$ in length, with a current pulse having an amplitude of $\approx 24 \mathrm{kA}$, a $10 \%$ to $90 \%$ rise time of $\approx 25 \mathrm{~ns}$ and a first halfcycle duration of $\approx 110 \mathrm{~ns}$. The discharge setup and pulse generator used in this experiment resemble those we described previously $[13,14]$. The fast current pulse was produced by discharging a water capacitor through a spark gap switch connected in series with the capillary load. The water served as a liquid dielectric for the capacitor and also cooled the capillary. The capacitor was pulse charged by a four-stage Marx generator that is enclosed in a separate box and connected to the laser head with a coaxial cable [11]. A nearly optimum Ar gas pressure of 490 mTorr was maintained in the capillary using a continuous Ar flow and a differential pumping system.

To determine the average output power the laser output pulse energy was measured for every shot using a vacuum photodiode placed at $87 \mathrm{~cm}$ from the exit of the laser. The data were recorded and stored by a $2 \times$
$10^{9}$ samples/sec digitizing oscilloscope with $500 \mathrm{MHz}$ analog bandwidth. The quantum efficiency of the Al photocathode was previously calibrated with respect to a silicon photodiode of known quantum yield [12]. The laser output was attenuated with several stainless steel meshes of measured transmissivity to avoid saturation of the photodiode. We have successfully operated the laser
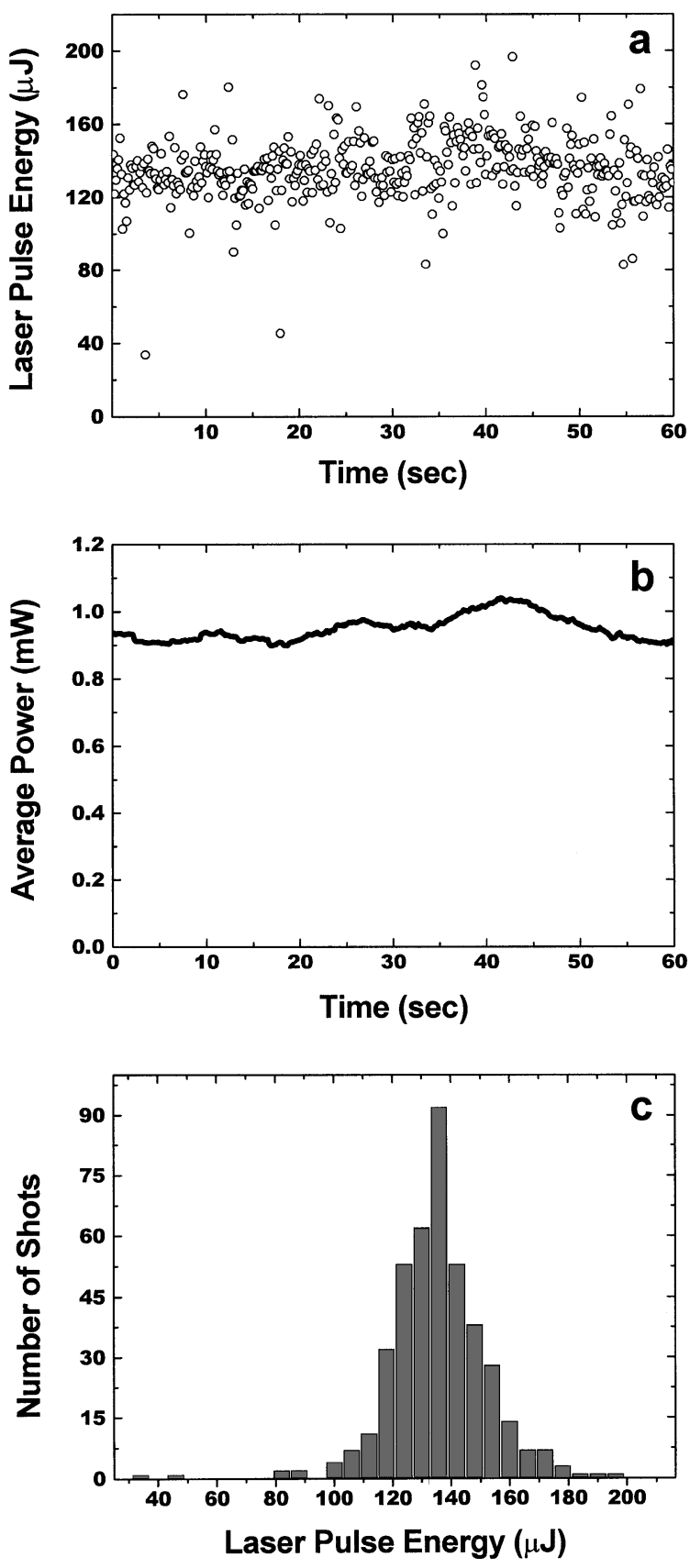

FIG. 1. Measured output pulse energy and average output power of the $46.9 \mathrm{~nm}$ laser corresponding to $1 \mathrm{~min}$ of continuous operation at a repetition rate of $7 \mathrm{~Hz}$. (a) Shot to shot laser output pulse energy. (b) Average output power computed as a running average of 50 contiguous laser pulses. (c) Distribution of the output pulse energy. The average pulse energy is $135 \mu \mathrm{J}$ and the standard deviation is $17 \mu \mathrm{J}$. 
at repetition frequencies up to $10 \mathrm{~Hz}$. However, the most detailed optimization was performed at $7 \mathrm{~Hz}$, resulting in the highest average power at this lower frequency. Figure 1 shows the measured laser output pulse energy and average power at $7 \mathrm{~Hz}$ repetition frequency at the near-optimum discharge pressure of 490 mTorr. The data correspond to $1 \mathrm{~min}$ of continuous operation of the laser. Figures 1(a) and 1(c) show that the average output energy per pulse is $(135 \pm 17) \mu \mathrm{J}$, and Fig. 1(b) shows that the average power at $7 \mathrm{~Hz}$ is $0.95 \mathrm{~mW}$. This corresponds to $>2.2 \times 10^{14}$ photons per second. We have operated the laser uninterruptedly during $30 \mathrm{~min}$ at $5 \mathrm{~Hz}$, and we have obtained up to $9 \times 10^{3}$ laser shots from a single capillary. After this number of shots lasing was still observed, but the output pulse energy degraded to about half its maximum value due to deterioration of the capillary walls.

The laser pulse width was measured using a vacuum photodiode and an analog oscilloscope with $1 \mathrm{GHz}$ bandwidth. The measurements were corrected by taking into account the limited frequency response of the oscilloscope. Figure 2 shows the temporal profile of a typical laser pulse. The full width at half maximum pulse width of the laser pulses generated at $7 \mathrm{~Hz}$ was measured to be $\approx 1.2 \mathrm{~ns}$. The laser pulse width was measured to be slightly larger at $1 \mathrm{~Hz}, \approx 1.3 \mathrm{~ns}$. The small reduction of the laser pulse width with increased repetition rate is most likely associated with a reduction of the density of Ar atoms in the capillary channel as a result of increased gas heating. This is supported by measurements that showed a decreased laser pulse width at reduced Ar pressures. The peak power of a typical laser pulse is $\approx 112 \mathrm{~kW}$, while the peak power of the most intense shots exceeds $150 \mathrm{~kW}$. The laser beam divergence and spatial intensity distribution were measured with a two-dimensional x-ray sensitive detector placed at $138 \mathrm{~cm}$ from the exit of the capillary. The detector consisted of a

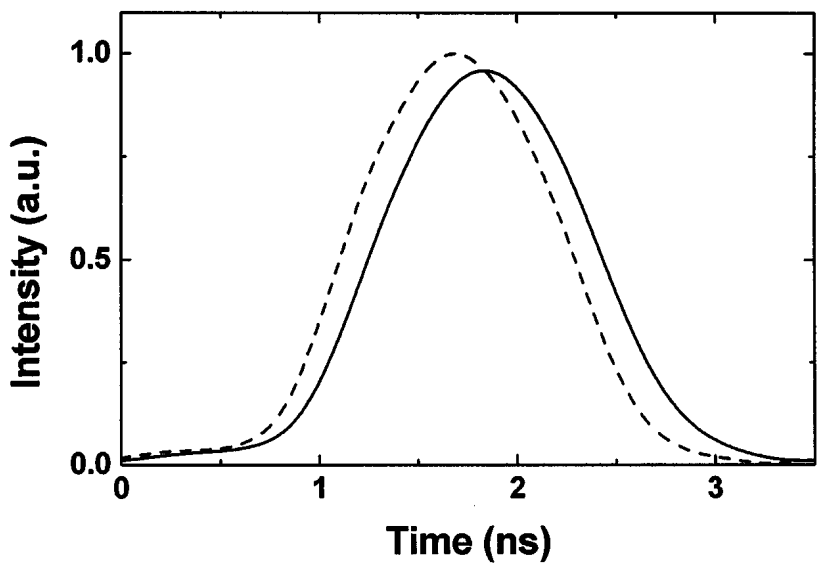

FIG. 2. Temporal evolution of the laser intensity for a typical laser shot generated at a repetition frequency of $7 \mathrm{~Hz}$. The solid curve is the photodiode signal recorded with a $1-\mathrm{GHz}-$ bandwidth analog oscilloscope. The dashed curve is the signal corrected for the limited bandwidth of the oscilloscope. phosphor screen and a charge-coupled device (CCD) array of $1024 \times 1024$ pixels. Figure 3 illustrates the measured spatial intensity distribution of the beam that has an annular shape. The annular shape is the result of refraction of the amplified rays in the plasma column due to a plasma density gradient in the radial direction [15]. The peak to peak beam divergence is $\approx 4 \mathrm{mrad}$.

It is of interest to compare the spatially coherent power emitted by this tabletop laser to that generated at $26.5 \mathrm{eV}$ in a similar bandwidth by a third-generation synchrotron beam line. For the purpose of making a comparison of output power per unit bandwidth, the laser linewidth can be conservatively estimated to be $\Delta \lambda / \lambda=1 \times 10^{-4}$. This results from considering that the Doppler broadened linewidth of the laser transition for an ion temperature of $T_{i} \approx 100 \mathrm{eV}$ [12] is about $\Delta \lambda / \lambda=1.2 \times 10^{-4}$, and that it narrows by a factor of $\left(g^{*} l_{\mathrm{sat}}\right)^{1 / 2} \approx 3.7$ as the radiation is amplified to reach saturation at $g^{*} l_{\text {sat }} \approx 14$ [12]. While some degree of line rebroadening might occur after saturation [16], the laser linewidth can be expected to still remain below $\Delta \lambda / \lambda=1 \times 10^{-4}$. Assuming that the spatial coherence of the laser beam produced in the ceramic capillary is similar to that recently measured for a discharge in a polyacetal capillary (about 6 times diffraction limited in the tangential direction and 8 to 9 times diffraction limited in the radial direction [17]), the average spatially coherent power can be estimated to be about $2 \%$ of the $0.95 \mathrm{~mW}$ measured, or $\approx 19 \mu \mathrm{W}$. This spatially coherent power is similar to the $\approx 20 \mu \mathrm{W}$ emitted at $\Delta \lambda / \lambda=1 \times 10^{-4}$ by the $8 \mathrm{~cm}$ period undulator at the Advanced Light Source,

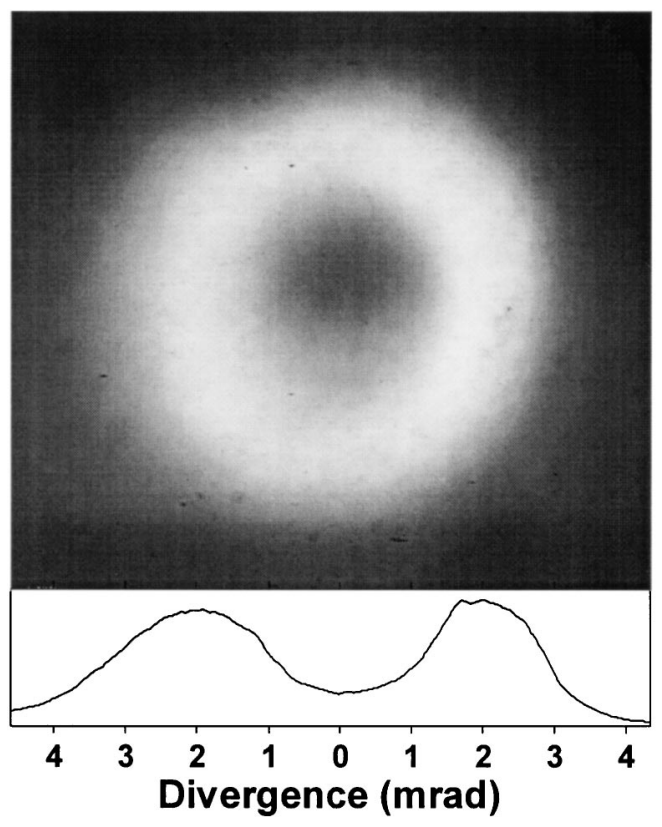

FIG. 3. Image of the laser beam at $1.38 \mathrm{~m}$ from the exit of the capillary. The intensity distribution corresponds to the sum of five consecutive shots at $7 \mathrm{~Hz}$. The annular beam pattern is caused by refraction. 
a third-generation synchrotron situated at Berkeley [18]. Nevertheless, for some applications the synchrotron has the advantage of broad tunability at shorter wavelengths. In contrast, the peak power of the spatially coherent radiation emitted by the capillary discharge laser is estimated to be about $2.3 \mathrm{~kW}$, and exceeds by more than 5 orders of magnitude that of the undulator, estimated at $\approx 4 \mathrm{~mW}$.

In conclusion, we have demonstrated the operation of a very compact tabletop $46.9 \mathrm{~nm}$ laser at an average power of $\approx 1 \mathrm{~mW}$. This result corresponds to an increase in the average power available from soft $\mathrm{x}$-ray lasers by 2 to 3 orders of magnitude. The demonstration of a tabletop soft x-ray laser with a spatially coherent average power per unit bandwidth similar to that of a synchrotron beam line has the potential to greatly expand the use of intense coherent short-wavelength radiation in applications.

We thank Vyacheslav Shlyaptsev and Siu Au Lee for many useful discussions and Elliot Bernstein for his interest and encouragement. We also acknowledge the assistance of Bill McHardy. This work was supported by the National Science Foundation under Grant No. DMR9512282.
[1] D. T. Attwood, K. Halbach, and N. J. Kim, Science 228, 1264 (1985).

[2] A. L'Huillier, in X-Ray Lasers 1996, edited by S. Svanberg and C.G. Wahlstrom (Institute of Physics, Bristol, 1996), p. 444.

[3] T. Ditmire et al., Phys. Rev. A 51, R902 (1995).

[4] A. Rundquist et al., Science 280, 1412 (1998).

[5] L. B. DaSilva et al., Opt. Lett. 18, 1174 (1993).

[6] P. V. Nickles et al., Phys. Rev. Lett. 78, 2748 (1997).

[7] J. Dunn et al., Phys. Rev. Lett. 80, 2825 (1998).

[8] B. E. Lemoff et al., Phys. Rev. Lett. 74, 1576 (1995).

[9] D. Korobkin, C. H. Nam, S. Suckewer, and A. Golstov, Phys. Rev. Lett. 77, 5206 (1996).

[10] D. Korobkin, A. Goltsov, A. Morozov, and S. Suckewer, Phys. Rev. Lett. 81, 1607 (1998).

[11] B. R. Benware, C. H. Moreno, D. J. Burd, and J. J. Rocca, Opt. Lett. 22, 796 (1997).

[12] J. J. Rocca et al., Phys. Rev. Lett. 73, 2192 (1994).

[13] J. J. Rocca, D. P. Clark, J. L. A. Chilla, and V. N. Shlyaptsev, Phys. Rev. Lett. 77, 1476 (1996).

[14] J. J. Rocca et al., Phys. Plasmas 2, 2547 (1995).

[15] C. H. Moreno et al., Phys. Rev. A 58, 1509 (1998).

[16] J. Koch et al., Phys. Rev. Lett. 68, 3291 (1992).

[17] M. C. Marconi et al., Phys. Rev. Lett. 79, 2799 (1997).

[18] D. T. Attwood et al., IEEE J. Quantum Electron. (to be published) (see Fig. 8, adjusted for $\Delta \lambda / \lambda=10^{-4}$ ). 